\title{
The Use of a Disclosing Agent During Resective Periodontal Surgery for Improved Removal of Biofilm
}

\author{
Marco Montevecchi, Vittorio Checchi, Maria Rosaria Gatto, Sascha Klein and Luigi Checchi
}

Department of Periodontology and Implantology, School of Dentistry, Alma Mater Studiorum - University of Bologna, Italy

\begin{abstract}
A total removal of the bacterial deposits is one of the main challenges of periodontal therapy. A surgical approach is sometimes required in order to allow a correct access to the areas not thoroughly reached during the initial therapy. The present study focuses on the surgical scaling effectiveness in root deposits removal; the potential support of a disclosing agent during this procedure is also evaluated. Forty surgical periodontal patients were randomly divided between surgeries where the operator was informed about a final examination of the residual root deposits and surgeries where the operator was not informed. Straight after scaling procedures a supervisor recorded the O'Leary Plaque Index of the exposed roots by mean of a disclosing agent and the percentage of teeth with residual biofilm. After the stained deposits removal, a second chromatic examination was performed and new data were collected. Mann-Whitney U-test and Wilcoxon test for paired samples were used for comparisons respectively between the two surgery groups and the first and the second chromatic examination; one-sided p-value was set at 0.05 . At first examination no significant differences between the two groups were observed regarding Plaque Index $(p=0.24)$ and percentages of teeth with residual biofilm $(\mathrm{p}=0.07)$. The $100 \%$ removal of roots deposits was never achieved during the study but a significant reduction of $80 \%$ of root deposits was observed between first and second examination $(\mathrm{p}=0.0001)$. Since root deposits removal during periodontal surgery resulted always suboptimal, the use of a disclosing agent during this procedure could be a useful and practical aid.
\end{abstract}

Keywords: Disclosing agent, biofilm, periodontal surgery, scaling and root planing.

\section{INTRODUCTION}

A "biologically healthy" root surface is considered a starting point for a natural and stable relationship between a tooth and its surrounding periodontal tissues [1]. Therefore the primary goal of an appropriate periodontal therapy should be to eliminate all the bacteria and their derivate products from the root surface [2].

A successful deposits removal depends upon correct management and understanding of multiple factors that can be grouped into the following three categories: instruments used, operator skills and visibility level.

Regarding instruments available, we assisted to a strong innovation surge over recent years. Miniaturized instruments with new forms, surfaces and materials are now commonplace within professional practice and the improved clinical results attained from their use have indeed lived up to expectations [3-6].

Besides many are the factors that could be related to operator skills. Among them some are inevitably subjective and extremely difficult to be controlled; others instead, even if operator dependent, are more related to the circumstances and, as a result, potentially more controllable. A particular

*Address correspondence to this author at the Department of Periodontology and Implantology, School of Dentistry, Alma Mater Studiorum, University of Bologna. Via S. Vitale 59, 40125 Bologna, Italy;

Tel: +39/0512080115; Fax: +39/0514391718;

E-mail: luigi.checchi@unibo.it psychological phenomenon called "Hawthorne Effect" [7] belongs to the last group; this effect refers to the tendency of some people to work harder and perform better when they are participants in an experiment. Persons may change their behavior due to the attention they are receiving from researchers rather than because of any manipulation of independent variables. This interesting variable should be considered each time the productivity of an operator is evaluated.

The last group of categories concerns the level of visibility of the clinician. Actually, the possibility to visualize the area of interest and thus its accessibility for cleansing has always been a crucial factor affecting the periodontist choice of treatment strategy [8]. The lack of visibility is often considered as a sufficient reason to decide for the surgical intervention. Though important technological innovations have undoubtedly enhanced the diagnostic-therapeutic potential of the clinician, a direct visual methodology is still deemed to be the best capable system of guaranteeing the highest removal percentage of bacterial deposits $[9,10]$. Apart from the obvious anatomical impediments associated with the visualization of subgingival areas, difficulties associated with the complete removal of bacterial deposits within the supragingival areas have also been previously demonstrated [11]. An extremely functional approach to this problem is the use of staining substances that adhere to the bacterial deposits on the tooth surfaces. The consequential improved visibility of the biofilm becomes a crucial factor for its simple and more effective removal. 


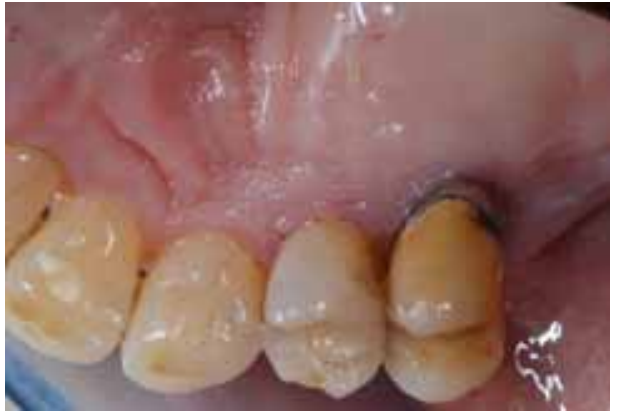

Fig. (1). Palatal view of the target area just before surgery.

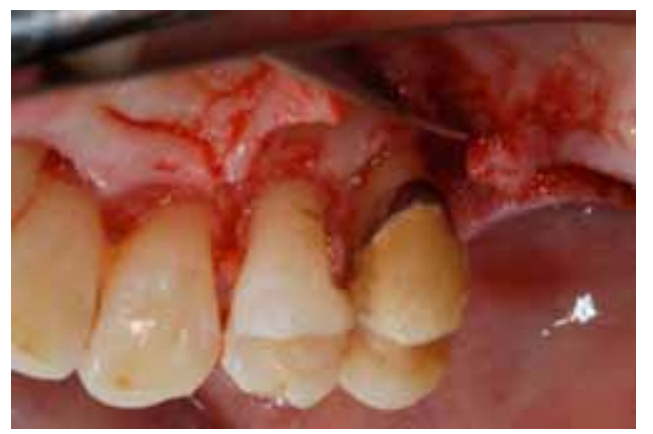

Fig. (2). Palatal view after flap rising: submarginal surfaces are now exposed and calculus is detectable.

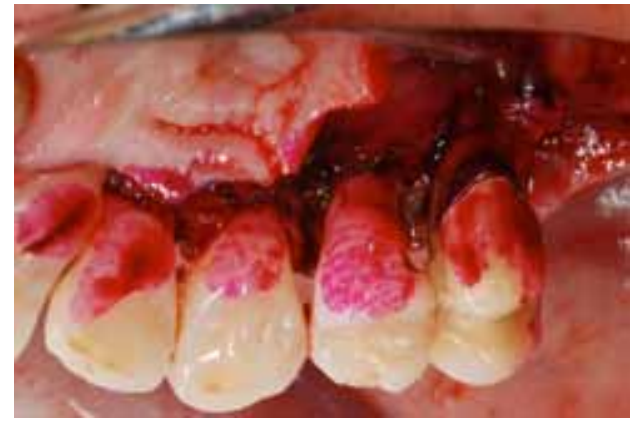

Fig. (3). Palatal view during disclosing agent application.

Aim of this study was to clinically evaluate the scaling effectiveness during osseous resective surgery and the potential aid of a disclosing agent during this procedure.

The influence on the scaling results induced by the operator awareness of a final supervision was also analyzed.

\section{MATERIALS AND METHODS}

From the patients attending the Department of Periodontology and Implantology of the "Alma Mater Studiorum" University of Bologna, a sample of 40 consecutive subjects fitting the following inclusion criteria was selected: presence of chronic periodontal disease in a moderate degree, necessity for the patient to undergo osseous resective periodontal surgery and absence of a declared allergy to any of the disclosing agent (Red-Cote ${ }^{\circledR}$, Butler) components. A periodontist not involved in the trial enrolled the participants.

In accordance with the principles of the Helsinki declaration of 1975, as revised in 2000, each patient enrolled in this study received a verbal and written explanation of the project and subsequently signed an informed consent form for participation.

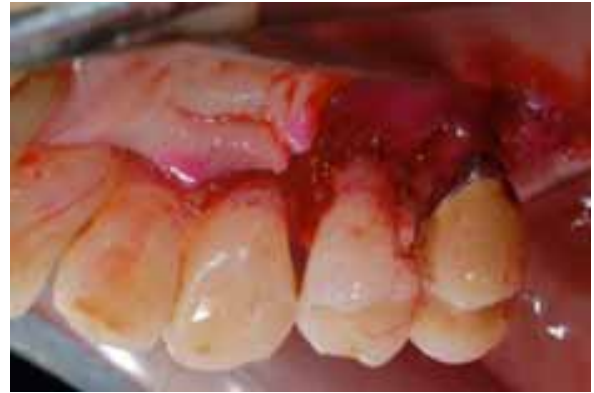

Fig. (4). Palatal view after disclosing agent washing: stained biofilm and calculus are now clearly detectable.

The surgical treatments (apically positioned flap with osseous resective surgery) were performed upon 20 patients where the operator was informed about the planned posttreatment chromatic examination by a supervisor and upon 20 patients where the operator was not previously informed about the examination. The patient allocation was randomized: numbers among 1 to 40 were inserted in a box and than extracted until every surgery was assigned. A pair number identified a patient treated by an informed operator and an odd number identified a patient treated by a not informed operator. The supervisor, an experienced periodontist, generated the allocation sequence and assigned the participants to their groups. Participants and operators were blinded to the group assignment.

In order to reduce the effects that operator fatigue and skill might have upon the study results, two operators with extensive periodontal experience performed all surgical interventions at the workday beginning. Following the necessary surgical exposure of the subgingival portions of the teeth, the periodontist proceeded, without any time limits imposed, with the scaling and root planing procedures using both ultrasonic and manual instruments. In cases in which the operator was informed about the planned controls, it was imposed that he communicate the moment in which the chromatic examination was to be carried out. In cases where the operator was not previously informed, the examination phase was performed just before suturing the flaps.

The chromatic examination, always carried out by the supervisor, was performed as follows: the stain $(1.5 \% \mathrm{w} / \mathrm{w}$, Food Red No. 104 in Japan, D\&C Red No. 28 in the USA) was applied in a passive manner using sterile swabs onto the exposed roots, left in situ for 10 seconds and then washed with sterile saline solution for an equal time.

The plaque index was assessed according to the O'Leary method [12] and the teeth percentage with residual biofilm was registered for each patient (the number of treated teeth with at least one stained area divided by the total number of treated teeth multiplied by one hundred).

After the removal of the stained deposits, a second chromatic examination was performed in order to verify if the use of the disclosing agent could be effective in improving the scaling results.

The lasting stains were finally cleaned away and the flap was sutured. Figs. (1 to 5) illustrate in sequence the most crucial steps of the technique. 


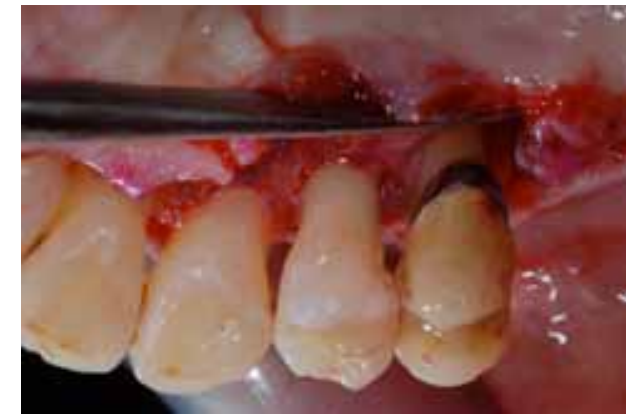

Fig. (5). Palatal view after root deposits removal.

Table 1. Mean Plaque Index (PI) at First and at Second Chromatic Examinations (p-Value: 0.0001)

\begin{tabular}{|c|c|c|}
\hline $\begin{array}{c}\text { Chromatic } \\
\text { Examination }\end{array}$ & Mean PI (\%) \pm Standard Error & Min - Max \\
\hline \hline First & $48.58 \pm 3.88$ & $20-96$ \\
\hline Second & $9.71 \pm 0.78$ & $4-19$ \\
\hline
\end{tabular}

\section{STATISTICAL ANALYSIS}

The sample dimension was defined as follows: a level of significance of 0.05 for a one-sided test and a power of $80 \%$ were considered, the $50 \%$ was judged as the absolute PI acceptable reduction between the two patient populations according to the clinical expectations. An intention-to-treat analysis was performed blind. The Kolmogorov-Smirnov Z test with the Lilliefors correction for significance was used to evaluate the fit of plaque index and percentage of teeth with residual biofilm to the Gaussian model. Consequently to the ascertainment of the not Gaussian distribution of the two parameters, the Mann-Whitney U-test was used to compare plaque index and percentage of teeth with residual biofilm between the two patient groups and the Wilcoxon test for paired samples was used to compare plaque index between the first and the second chromatic examination. One-sided p-value at 0.05 was considered on the basis of the research hypothesis that was a positive influence on the surgical scaling result by the consciousness of a final supervision [13]. Statistical assessment of the differences between frequencies of cleaned surfaces (lingual, vestibular, medial, distal) was performed using the $\chi^{2}$ test; the level of significance adjusted for multiple comparisons by using the Bonferroni method was 0.008 .

\section{RESULTS}

The 40 patients forming the study sample, recruited from July to December 2008, were $56 \%$ females and $44 \%$ males with a mean age $51 \pm 7$ years, ranging from 36 to 66 . All the participants completed the study protocol.
No complications occurred in any patients, neither during nor after surgery.

A significant reduction $(80 \%, \mathrm{p}=0.0001)$ of the PI score between the first and the second chromatic examination was observed [Table 1].

The first chromatic examination revealed that a $100 \%$ removal of the bacterial deposits was never achieved. Distribution and number (\%) of dental areas with identified deposits were as follow: 130 lingual areas (31\%); 124 distal areas $(29.5 \%), 92$ medial areas $(21.9 \%), 74$ vestibular areas $(17.6 \%)$. At this examination, the lingual and distal areas were significantly more stained than the vestibular ones $(\mathrm{p}=0.001)$ as the lingual areas were compared to the medial ones $(\mathrm{p}=0.003)$.

Informing or not the operator regarding the posttreatment examinations did not result as being significant upon the distribution of the areas that remained unclean.

The plaque index did not differ in a statistically significant manner between the interventions in which the periodontist was informed about the final examination and those in which the operator was not informed [Table 2].

In all cases, at least 2 teeth showed residues of biofilm at the moment of suturing. Between the two groups of surgeries, the percentage of teeth with residual biofilm did not differ in a statistically significant manner but tended to because of $\mathrm{p}=0.07$ [Table 2].

\section{DISCUSSION}

In the present study, the complete removal of biofilm residues on root surfaces during osseous resective periodontal surgery with apically positioned flap was never achieved. Moreover, informing the operator about a final supervision of the cleansing procedures performed during the surgery did not significantly influence the quality of the results. The last finding suggests that the precision in the cleansing process does not probably relate to the Hawthorne Effect. The absence of an evident role of this psychological phenomenon could be explained in two ways: one reason could be a negligible role of it in this specific procedure and the second reason could be the eventual presence of a major limiting factor.

Interestingly, the use of a disclosing agent during periodontal resective surgery seems to be instead effective in improving the scaling and root planing results.

This finding, associated to the observation that at the first chromatic examination the distal and lingual areas remained more frequently unclean than the vestibular areas, suggests that accessibility and visibility are important limiting factors in determining the quality of the cleansing outcome.

Table 2. Mean Plaque Index (PI) and Percentage of Teeth with Residual Plaque Related to the Operator's Awareness about the PostTreatment Chromatic Examination

\begin{tabular}{|c|c|c|c|}
\hline $\begin{array}{c}\text { Operator } \\
\text { Awareness }\end{array}$ & $\begin{array}{c}\text { Surgery } \\
\text { Numbers }\end{array}$ & Mean PI $(\boldsymbol{\%}) \pm$ Standard Error & Mean (\%) of Teeth with Plaque Residues \pm Standard Error \\
\hline \hline Yes & 20 & $41.90 \pm 4.61$ & $84.2 \pm 8.0$ \\
\hline No & 20 & $55.26 \pm 5.99$ & $92.4 \pm 6.0$ \\
\hline
\end{tabular}




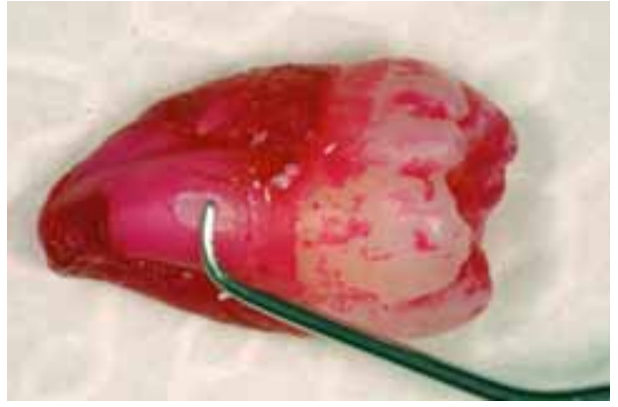

Fig. (6). Third molar, devoid of periodontal problems, extracted and stained according to the protocol of this study. Note the pigmented periodontal ligament and the area free of stain after a curette stroke.

Among the clinical variables, the absence of a marked chromatic contrast between the bacterial deposits and the dental tissues is probably one of the major limitations.

What are the effective consequences of such a limit in the total removal of the root deposits is not known and is beyond the scope of this study. A certain tissue tolerance is undoubtedly present; indeed, it has been shown that the periodontal tissues are also able to respond favorably to surfaces that remain partially contaminated [14]. Unfortunately the amount of residual deposits, as threshold on tissue tolerance, is still far from being defined. Furthermore, it must be firmly upholded that the variability in tissue response is inevitably subject to many factors, both inherent and external to the individual, that in turn makes the characterization of such a threshold truly complex [15]. To recapitulate, all causal factors should be systematically removed during the surgical phases of treatment and all procedures that might contribute to successfully attain the desired clinical results should be considered. From the results of the present study it derives the suggestion that the plaque disclosing agent, as for the supragingival region, should also constitute a useful, practical and economical aid for the periodontist during surgical scaling [Table 1]. Numerous in vivo and in vitro studies have been carried out on such plaque disclosing agents but no study has yet investigated their use during periodontal surgery [16-21].

The disclosing agent used in the present study contains Phloxine B as the staining substance, a widely used color additive for food, drugs, and cosmetics. Few is known about the potential toxicity of this colorant but a certain teratogenicity has been observed on mice after a 10 days of continuative food somministration [22], however no adverse effects have ever been reported during oral conventional use. Hence, the application of it during a surgical exposure in a controlled and limited quantity with commercial concentration $(1.5 \% \mathrm{w} / \mathrm{w})$ should be considered a safe procedure.

Preparatory to the present study, it was necessary to evaluate, on freshly extracted teeth, the performance of the plaque disclosing agent on periodontal ligament. As shown in Fig. (6), erythrosine, applied following the described protocol, binds ubiquitously to the root surfaces presenting an intact periodontal ligament. This observation brings about the proposal that such protocols should only be considered for resective surgery. The pigmentation of such tissue portions could bring to a clinical discerning error regarding the residual deposits and as a result to an unintentional but "extremely harmful" removal of tissues thought to be important for reattaching and/or regenerative processes [23].

\section{CONCLUSIONS}

In conclusion this study shows that a total removal of root deposits during an osseous resective surgery is never obtained with conventional instrumentation. Clinical limitations and visual deficiency could be the primary responsible of the present results. In order to overcome these obstacles, the use of a plaque disclosing agent during resective surgeries seems to be effective.

Though this observation, the post-operative clinical parameters remain to be assessed in order to evaluate the attainable advantages that affect both recovery phases and long-term periodontal health. Such observations would constitute an interesting starting point for future experimental studies on this subject.

\section{ACKNOWLEDGEMENT}

No external funding, apart from the support of Authors institution, was available for this study.

\section{CONFLICT OF INTEREST}

There was no conflict of interest.

\section{REFERENCES}

[1] Anderson GB, Palmer JA, Bye FL, Smith BA, Caffesse RG. Effectiveness of subgingival scaling and root planing: single versus multiple episodes of instrumentation. J Periodontol 1996; 67: 367-73.

[2] Greenstein G. Periodontal response to mechanical and non-surgical therapy: a review. J Periodontol 1992; 63: 118-30.

[3] Otero-Cagide FJ, Long BA. Comparative in vitro effectiveness of closed root debridement with fine instruments on specific areas of mandibular first molar furcations. I. root trunk and furcation entrance. J Periodontol 1997; 68: 1093-7.

[4] Otero-Cagide FJ, Long BA. Comparative in vitro effectiveness of closed root debridement with fine instruments on specific areas of mandibular first molar furcations. II. furcation area. J Periodontol 1997; 68: 1098-101.

[5] Barendregt DS, Van der Velden U, Timmerman MF, Van der Weijden F. Penetration depths with an ultrasonic mini insert compared with a conventional curette in patients with periodontitis and in periodontal maintenance. J Clin Periodontol 2008; 35: 31-6.

[6] Arabaci T, Ciçek Y, Canakçi CF. Sonic and ultrasonic scalers in periodontal treatment: a review. Int J Dent Hyg 2007; 5: 2-12.

[7] McCarney R, Warner J, Iliffe S, van Haselen R, Griffin M, Fisher P. The hawthorne effect: a randomised, controlled trial. BMC Med Res Methodol 2007; 3(7): 30.

[8] World workshop in periodontics 1996. Chicago: American academy of periodontology. Ann of Periodontol 1996; 1: 1-947.

[9] Kasaj A, Moschos I, Röhrig B, Willershausen B. The effectiveness of a novel optical probe in subgingival calculus detection. Int $\mathbf{J}$ Dent Hyg 2008; 6: 143-7.

[10] Michaud RM, Schoolfield J, Mellonig JT, Mealey BL. The efficacy of subgingival calculus removal with endoscopy aided scaling and root planing: a study on multirooted teeth. J Peridontol 2007; 78 : 2238-45.

[11] Checchi L, Forteleoni G, Pelliccioni GA, Loriga G. Plaque removal with variable instrumentation. J Clin Periodontol 1997; 24: 715-7.

[12] O'Leary TJ, Drake RB, Naylor JE. The plaque control record. J Periodontol 1972; 43: 38.

[13] Daniel WW. Naples: Ed; Edi Ses: Biostatistica1996.

[14] Robertson PB. The residual calculus paradox. J Periodontol 1990; 61: 65-6.

[15] Scapoli C, Mamolini E, Trombelli L. Role of IL-6, TNF-A and LTA variants in the modulation of the clinical expression of plaqueinduced gingivitis. J Clin Periodontol 2007; 34: 1031-8. 
[16] Gallagher IH, Fussell SJ, Cutress TW. Mechanism of action of a two-tone plaque disclosing agent. J Periodontol 1997; 48: 395-6.

[17] Kipioti A, Tsamis A, Mitsis F. Disclosing agents in plaque control. evaluation of their role during periodontal treatment. Clin Prev Dent 1984; 6: 9-13.

[18] Fujikawa K, Sugai K, Suzuki K, Haruta K, Okada A, Murai S. A comparison of the effect of various plaque disclosing materials on plaque accumulation (in Japanese). Nippon Shishubyo Gakkai Kaishi 1983; 25: 399-404.

[19] Schneider HG, Friedlander B, Richter CH, Unger E, Ziesenitz S, Bendrien S. Experience with plaque-disclosing agents (in German). Stomatol DDR 1981; 31: 904-9.
[20] Tan AE, Wade AB. The role of visual feedback by a disclosing agent in plaque control. J Clin Periodontol 1980; 7: 140-8.

[21] Cohen DW, Stoller NH, Chace R, Laster L. A comparison of bacterial plaque disclosants in periodontal disease. J Periodontol 1972; 43: 333-8.

[22] Seno M, Fukuda S, Umisa H. A teratogenicity study of Phloxine B in ICR mice. Food Chem Toxicol 1984; 22: 55-6.

[23] Polimeni G, Xiropaidis AV, Wikejö UM. Biology and principles of periodontal wound healing/regeneration. Periodontol 2000 2006; 41: 30-47.

(C) Montevecchi et al.; Licensee Bentham Open.

This is an open access article licensed under the terms of the Creative Commons Attribution Non-Commercial License (http://creativecommons.org/licenses/by-nc/3.0/) which permits unrestricted, non-commercial use, distribution and reproduction in any medium, provided the work is properly cited. 